\title{
Meta
}

Journal des traducteurs

Translators' Journal

Branchadell, A. and L. M. West (eds) (2005) : Less Translated

Languages, Amsterdam/Philadelphia, John Benjamins

Publishing Company, Collection Benjamins Translation

Lilbrary, volume 58, VIII + $414 \mathrm{p}$.

\section{Serge Marcoux}

Volume 52, numéro 3, septembre 2007

URI : https://id.erudit.org/iderudit/016743ar

DOI : https://doi.org/10.7202/016743ar

Aller au sommaire du numéro

Éditeur(s)

Les Presses de l'Université de Montréal

ISSN

0026-0452 (imprimé)

1492-1421 (numérique)

Découvrir la revue

Citer ce compte rendu

Marcoux, S. (2007). Compte rendu de [Branchadell, A. and L. M. West (eds) (2005) : Less Translated Languages, Amsterdam/Philadelphia, John Benjamins Publishing Company, Collection Benjamins Translation Library, volume 58, vII + 414 p.] Meta, 52(3), 577-581. https://doi.org/10.7202/016743ar d'utilisation que vous pouvez consulter en ligne.

https://apropos.erudit.org/fr/usagers/politique-dutilisation/ 
Branchadell, A. and L. M. West (eds) (2005): Less Translated Languages, Amsterdam/ Philadelphia, John Benjamins Publishing Company, Collection Benjamins Translation Library, volume 58, viI +414 p.

Fruit des travaux de la $5^{e}$ Conférence internationale sur la traduction tenue en 2001, Less Translated Languages se veut une analyse méthodique de ce concept calqué sur celui des «langues peu utilisées». Le catalan, langue qui a connu au cours des siècles diverses périodes d'effacement et de renaissance, est au cœur de cette analyse et constitue un excellent exemple de l'adaptation que doit faire une langue longtemps tenue dans l'ombre pour s'adapter aux réalités du monde moderne.

La première partie du livre est consacrée au phénomène de l'anglais comme langue dominante de notre époque.

Une première contribution met en garde contre la tentation de voir dans le fait que plus de traductions sont effectuées à partir de l'anglais que vers cette langue un sombre complot de la part de maisons d'édition ou une volonté d'impérialisme culturel. Anthony Pym et Grzegorz Chrupała démontrent grâce à un modèle mathématique qu'il est dans l'ordre des choses que la langue dans laquelle sont publiés le plus grand nombre de livres soit aussi celle à partir de laquelle se fassent le plus grand nombre de traductions; à l'inverse, moindre est le nombre de livres publiés dans une langue, moindre également sera le nombre de traductions faites à partir de la même langue. S'y ajoute le fait que plus d'un lecteur bilingue ou multilingue préfere lire la version originale d'une œuvre, donnant ainsi un avantage supplémentaire à l'anglais.

Par opposition à cette prédominance de fait, l'Union européenne affirme l'égalité de droit entre les 23 langues officielles de ses pays membres ${ }^{1}$, égalité poussée si loin que les traductions des textes législatifs ne sont pas considérées comme des traductions, mais bien comme des versions originales puisque le texte source, dans quelque langue qu'il ait été rédigé, cesse d'exister une fois traduit. Sur le plan de la forme, les textes doivent se présenter de façon identique et contenir le même nombre d'articles, de paragraphes et même de phrases. Vilelmini Sosonis nous rappelle toutefois les limites de cette égalité de droit: ne sont considérées langues de la communauté que les langues officielles des États. De telle sorte que les parlants français d'Italie ne pourront pas nécessairement communiquer dans cette langue avec les autorités de l'Union, pas plus que les parlants castillan d'Espagne. Pardoxalement, cette multiplication des langues officielles renforce la prééminence de l'anglais, du français et de l'allemand dans la vie quotidienne de la communauté aux dépens de langues moins fréquemment utilisées et traduites comme le danois ou le grec.

L'une des raisons pour laquelle certaines langues sont moins traduites que d'autres réside dans la difficulté qui leur est propre de s'adapter aux rapides changements scientifiques et technologiques que connait notre époque. Partant du fait que, dans les langues occidentales, ce vocabulaire scientifique se forme souvent par l'ajout de préfixes et de suffixes d'origine gréco-latine, Hassan Hamzé étudie la possibilité d'élaborer un système similaire pour l'arabe afin de simplifier et d'accélérer la traduction. Mais, outre que le système n'est pas identique entre toutes les langues européennes (le français et l'allemand par exemple), les différences morphologiques fondamentales entre ces langues et l'arabe rendraient pratiquement impossible la création d'un système de dérivation copié sur celui du français.

Si elles ont tendance à "occulter» les langues minoritaires, les langues dominantes peuvent servir de langues intermédiaires au moment du passage vers diverses langues moins souvent traduites. Ainsi, Nobel Pedu Honeyman explique comment la traduction en anglais du Kitáb-i-Aqdas, le livre saint de la religion Bahá'í, a permis de réaliser, en moins d'un an, une nouvelle traduction, cette fois en espagnol, à l'intention des hispanophones d'Europe et d'Amérique. Même si le procédé n'a guère la faveur de la communauté scientifique, l'auteur estime qu'il peut s'avérer fort utile à l'occasion. Outre des économies de coûts appréciables, 
il permet d'établir une unité de format et de présentation pour des langues cibles qui partagent une même culture lorsque celle-ci est éloignée de celle de la langue source (dans l'exemple cité en allant du persan vers l'espagnol, le français et autres langues occidentales par l'entremise de l'anglais).

Toutefois, l'utilisation généralisée de l'anglais comme langue intermédiaire sous l'influence de la mondialisation peut rendre difficile le passage des références culturelles d'une langue à l'autre. Utilisant comme exemple la technique du sous-titrage au cinéma, et se basant sur une typologie en neuf catégories, Maria D. Oltra Ripoll propose de rendre ces données culturelles perceptibles au spectateur grâce à diverses techniques, allant du simple transfert (le mot russe perestroika utilisé sans variante dans d'autres langues) à la substitution par une autre référence culturelle qui peut être mieux comprise dans la culture d'arrivée, selon la flexibilité de cette dernière, de ses politiques linguistiques ainsi que de modèles linguistiques, stylistiques et culturels qui permettent une «traduisibilité ${ }^{»}$ de l'une vers l'autre.

La deuxième partie du volume traite des langues minoritaires sous l'angle de leur inégalité dans les politiques de traduction en vigueur.

Oscar Diaz Fouces distingue d'abord trois niveaux de langues à l'intérieur de l'Union européenne: les langues officielles des États-membres, qui sont aussi langues officielles de l'Union, les langues des minorités à l'intérieur des États-membres comme le catalan, le breton ou le gaélique, lesquelles ne jouissent au mieux que d'une reconnaissance partielle, et les langues des populations immigrées au sein des États-membres qui ont un statut officiel quelque part ailleurs au monde (ainsi, les Vietnamiens, s'ils ne peuvent trouver d'étiquetage dans leur langue sur les boîtes de conserve achetées en France, peuvent en trouver facilement au Vietnam, alors que les Bretons n'en trouveront ni en France, ni ailleurs). Se basant sur les critères d'homogénéité, d'autonomie et de prestige, il propose que l'on vienne en aide aux communautés de la deuxième catégorie, dites minorisées, par l'adoption d'une politique de traduction davantage proactive que celle qui a cours à l'heure actuelle.

Étudiant le phénomène de la traduction des langues minoritaires dans des communautés bilingues ou multilingues, Marta García González souligne que la traduction ne répond pas seulement à un besoin de communication, mais aussi à différents impératifs politiques, commerciaux ou autres tant entre langues minoritaires et majoritaires qu'entre langues minoritaires elles-mêmes. Sur cette base, elle tente d'élaborer un modèle qui permette de mesurer l'influence des divers facteurs sociaux qui incitent à traduire des textes vers ou en provenance de ces langues et l'influence qu'auront ces traductions sur leur normalisation.

Dans certains pays, la traduction vers une langue minoritaire est obligatoire là où elle est parlée par un grand nombre d'habitants. Albert Branchadell définit trois niveaux de traduction obligatoire: les cas où la traduction n'est jamais obligatoire, les cas où la traduction est nécessaire dans certains cas seulement et ceux où elle s'impose toujours. En théorie, l'obligation de traduire vers les langues minoritaires a pour but d'en assurer la pérennité, mais des considérations de temps et d'argent peuvent faire que ce but n'est pas toujours atteint.

Dans une contribution intitulée "Theatre and Translation: Unequal Exchanges in a Supermarket of Cultures», Eva Esposa met en lumière les limites du dialogue interculturel et, partant, l'impossibilité pour le traducteur de transposer ou de «réfracter» une culture autrement qu'en se l'appropriant ou se l'expropriant. Toutefois, cela est le fait de toutes les langues et ne s'applique pas seulement aux traductions faites entre langues majoritaires et langues minoritaires puisque, dans certains cas, elles s'avéreront facteurs d'assimilation et, dans d'autres, d'assertion d'une identité culturelle distincte comme au Québec ou en Écosse.

Enfin, le dernier chapitre (qui serait peut-être plus à sa place au début de la troisième partie) traite des «cultures occultées». Pour Anna Aguilar-Amat et Jean-Bosco Botsho, la 
traduction doit être essentiellement «respect de la différence», reconnaissance du fait que l'altérité repose sur des paradigmes différents d'une culture à l'autre et non de supériorité ou d'infériorité. Ainsi, la littérature africaine doit être jugée en fonction de critères africains et non de critères européens. Ce faisant, on découvrira que si les auteurs africains peuvent «communiquer» avec leur public en anglais, ils ne peuvent vraiment exprimer la totalité de leur culture dans cette langue qui devient ainsi pour l'élite un instrument de domination.

Nous en arrivons ainsi à la troisième partie qui traite des traductions faites à partir de langues minorisées ou occultées vers les langues européennes.

Analysant la littérature africaine écrite dans une «langue coloniale», Goretti López Heredia postule que la traduction des créations littéraires d'auteurs africains qui s'expriment en français, anglais, espagnol ou portugais correspond à la traduction de «silences». On découvre par ces œuvres comment certaines cultures ont été réduites au silence, tenues à l'écart et méconnues par l'impérialisme. Le phénomène postcolonial a tracé une ligne de démarcation entre les littératures européennes rédigées dans la langue des colonisateurs et ses contreparties colonisées, réduites dès lors à un rôle subalterne.

De façon moins politique, Andrés Xoxé Salter Iglesias illustre ce phénomène en disséquant les difficultés auxquelles se heurte le traducteur espagnol du livre Vozes Anoitecidas de l'auteur mozambicain Mia Couto, difficultés culturelles plus que linguistiques si l'on tient compte des similarités lexicales et syntaxiques existant entre l'espagnol et le portugais. La réalité sociale de la culture mozambicaine prend forme dans des tournures linguistiques et des néologismes soigneusement choisis par Couto pour définir l'identité de sa terre natale. La difficulté pour le traducteur consiste à trouver un compromis entre une reconstitution si fidèle de ces tournures qu'elles deviennent inintelligibles pour le lecteur et une adaptation si poussée à la culture d'arrivée qu'elle efface toute trace d'exotisme.

Dora Sales Salvador aborde le même problème, mais dans le contexte multilingue de l'Inde où l'hindi est reconnu comme langue officielle, l'anglais comme langue officielle associée et un grand nombre de langues régionales comme «langues nationales». Tout en reconnaissant les pertes ou les concessions qu'impose l'utilisation d'une langue autre que la langue maternelle aux auteurs indiens s'exprimant en anglais, elle illustre au moyen de textes empruntés à trois auteurs les gains que peut rapporter une telle production sur le plan de la communication entre les langues, les cultures et les sensibilités esthétiques. Pour elle, les différences ne peuvent et ne doivent pas être «occultées», mais plutôt «négociées».

C'est aussi la conclusion à laquelle aboutit Nicole Mart nez Melis dans son analyse des différentes traductions du traité bouddhiste Bodhicary vat ra faites à partir du sanskrit et du tibétain. Ce qui importe ici n'est pas de préserver une langue morte, le sanskrit, mais la tradition qu'elle symbolise. À partir de six traductions différentes de ce livre saint, l'auteur montre comment les premières traductions, datant du début du $\mathrm{xx}^{\mathrm{e}}$ siècle, avaient un caractère de recherche universitaire et visaient essentiellement à donner accès à un texte ancien; avec le début des années 1970, les traductions sont devenues le fait de bouddhistes pratiquants qui y voient une source d'inspiration. Ces deux approches sont en contradiction formelle avec la thèse exposée ailleurs dans le livre, selon laquelle l'Occident aurait continuellement cherché à «occulter» les langues minorisées des pays colonisés.

De la même façon, Letitcia Herrero, recensant la littérature régionale indienne publiée en anglais, affirme qu'il y a place dans les grandes maisons d'édition pour des traductions de textes écrits dans des langues ignorées à ce jour. Prenant appui sur un projet mis en œuvre par la filiale indienne d'une grande maison d'édition britannique, elle compare les aspects positifs et négatifs de la traduction d'une ouvre écrite en tamoul, Lamps in the Whirlpool. Elle reconnait sans peine toutefois que l'accumulation de notes de bas de page et la longue introduction à la culture tamoule placée au début du livre peuvent exaspérer certains lecteurs lorsque les réalités qu'elles décrivent sont si éloignées des références culturelles occidentales qu'elles demeurent impénétrables. 
Sara Rovira-Esteva aborde un problème similaire, mais non limité aux langues peu traduites: comment rendre dans la langue cible des catégories qui existent dans la langue source mais non dans la langue d'arrivée? Ainsi, à côté de catégories grammaticales proprement dites, la langue chinoise utilise diverses métaphores pour créer de nouvelles catégories qui classent les noms d'après leur apparence, leur volume, etc. Il y a aura donc une expression pour désigner une [paire de] baguette[s] et une autre pour désigner [une entité longue et mince] de baguettes. À défaut de rendre ces catégories, le traducteur s'expose à omettre un aspect de la réalité qui peut, à l'occasion, s'avérer importante.

La dernière partie du livre est tout entière consacrée au catalan comme langue de traduction.

Dans un premier chapitre, Montserrat Bacardi retrace l'évolution de la langue catalane, soulignant que pendant plusieurs siècles, il était nécessaire de faire la traduction d'une langue à l'autre dans la péninsule ibérique où les diverses langues régionales se sont souvent développées par opposition l'une à l'autre. Ainsi, si le $\mathrm{XvI}^{\mathrm{e}}$ siècle a vu l'essor de la littérature espagnole, il a aussi marqué le déclin du catalan. Ce ne fut qu'au XIX ${ }^{\mathrm{e}}$ siècle, alors que le castillan retrouva ses lettres de noblesse culturelles et sociales, que l'on commença à voir réapparaître des traductions de l'espagnol vers le catalan. Après une longue période d'absence imposée par le régime Franco, le catalan est redevenu une langue à part entière et les écrivains qui s'expriment dans cette langue peuvent espérer voir leurs œuvres traduites en espagnol.

Dans le chapitre suivant, Cristina García de Toro fait le point sur la traduction entre l'espagnol et le catalan. Sur le plan littéraire, à l'exclusion de la littérature pour la jeunesse, il se fait plus de traductions du catalan vers l'espagnol que l'inverse, sans doute parce que, comme au cinéma, le public bilingue préfère lire (ou voir) les œuvres en langue originale. De plus, plusieurs séries télévisées originellement en langue catalane ont eu un succès tel qu'elles ont dû être traduites en espagnol pour être également diffusées en Amérique du Sud. Sur le plan administratif, les deux langues ont statut officiel en Catalogne de telle sorte que non seulement les textes de l'État espagnol, mais aussi ceux de l'Union européenne doivent être traduits en catalan. La tendance s'inverse sur le plan juridique où, en dépit des efforts du gouvernement régional, peu de causes sont plaidées en catalan. Enfin, sur le plan scientifique, on note une augmentation des manuels et ouvrages publiés en catalan grâce à la création d'un Centre de terminologie catalane en 1985.

Pour compléter ce tour d'horizon, Irene Llop Jordana traite des traductions entre deux langues minoritaires, l'hébreu et le catalan. Après avoir dressé une liste des traducteurs les plus importants, elle décrit l'état des études hébraïques en Catalogne, énumérant les principales difficultés que pose la traduction entre ces deux langues avant de tracer un tableau historique des grandes étapes de ces traductions, en partant de la Bible pour en arriver à la littérature contemporaine.

La deuxième section de cette quatrième partie est le résultat d'un symposium tenu sur six personnalités s'étant consacrées à la traduction d'œuvres composées dans des langues dominantes (français, anglais, italien, etc.) vers le catalan.

La première contribution, rédigée par Judit Figuerola, est consacrée à Andreu Nin (18921937), successivement activiste politique, enseignant, journaliste, critique littéraire et traducteur. Engagé dans un parti politique trotskiste local, Nin fera l'objet d'attaques virulentes aussi bien de la part des partis de droite que du parti communiste. Après l'interdiction de ce parti par le gouvernement Negrín, Nin sera arrêté et disparaitra, probablement assassiné par la police politique soviétique qui opérait dans l'Espagne républicaine. On lui doit d'avoir fait connaître au public catalan plusieurs grands maîtres de la littérature russe, comme Dostoïevski, Tolstoï et Tchékhov.

Judit Fontcuberta i Famadas nous présente pour sa part un traducteur prolifique qui travailla à la renaissance de la langue catalane à une époque où l'Espagne demeurait en marge des grands courants de pensée internationaux. Né en 1899, Bonaventura Vallespinosa fut 
médecin de profession. Grand amateur de théâtre, il traduisit de nombreuses pièces, en particulier d'auteurs français comme Sartre et Camus, auxquels il vouait une grande admiration. Président du Centre de lecture de Reus, il assura la production et la mise en scène de plusieurs de ces pièces. Bien qu'il ait travaillé surtout à partir du français et de l'italien, Vallespinosa traduisit également des auteurs états-uniens comme Tennessee Williams afin de permettre au public catalan d'accéder aux grandes œuvres contemporaines.

Un autre traducteur et écrivain prolifique fut Manuel de Pedrolo auquel est consacrée la contribution de Alba Pijuan Vallverdú. Manuel de Pedrolo faisait partie d'une génération qui n'avait pu apprendre le catalan à l'école, pas plus qu'elle ne pouvait le lire ou l'entendre dans les médias. Il lui incomba donc, avec les autres traducteurs qu'il put réunir dans les Edicions 62, de fixer des normes et de standardiser la langue d'une part, d'intéresser un public aux œuvres écrites dans cette langue d'autre part, ce qu'il fit à la fois pour la poésie, le roman de style américain, le théâtre existentialiste et celui de l'absurde, ainsi que pour le roman policier, genre qu'il créa littéralement en catalan.

Comme ses contemporains, Josep Vallverdú commença à écrire et à publier en espagnol. Comme nous l'apprend Anna Cris Mora, ce n'est qu'avec la création des Edicions 62 et l'assouplissement du régime Franco que put débuter une période de production et de traduction littéraire en catalan. Tout comme de Pedrolo, Vallverdú s'intéressa au roman policier qui donnait l'occasion de créer un langage de la rue dans lequel on pourrait reconnaître le vocabulaire propre aux voleurs et aux assassins. En 1966, il organisa la première table ronde où les traducteurs catalans purent vraiment discuter des difficultés de la traduction dans une langue qui émergeait d'une longue période d'obscurité.

Avec Maria-Mercè Marçal, débute une nouvelle génération de traducteurs dont les champs d'intérêt se démarqueront de ceux de leurs prédécesseurs. Militante féministe, membre de la classe ouvrière et citoyenne d'un pays en proie à la dictature, celle-ci s'attachera à l'œuvre d'autres femmes ayant fait œuvre de précurseures comme Marguerite Yourcenar, Colette, Leonor Fini, Monika Zgustová, Anna Akhmatova et Marina Tsetayeva. Dans son analyse, Pilar Godayol s'applique à nous montrer comment la traduction (re)produit et (re)présente l'œuvre originale par ses additions et soustractions, sa contamination par le style propre du traducteur et sa conceptualisation de l'œuvre dans la langue d'arrivée.

Cette galerie de portraits se termine par la contribution de Victória Alsina Keith consacrée à Jordi Arbonès i Montull. Né en 1929 en pays catalan, autodidacte, il passa la plus grande partie de sa vie en Argentine où il dirigea la librairie catalane, espace littéraire et lieu d'expositions, de conférences et de pièces de théâtre. Fruit de cette synthèse de deux continents et traducteur prolifique (il traduisit pas moins de 93 livres en catalan et 48 en espagnol), il fut l'un des premiers à faire connaître les auteurs dramatiques états-uniens des $\mathrm{XIX}^{\mathrm{e}}$ et $\mathrm{XX}^{\mathrm{e}}$ siècle au public catalan. Quoique certains de ses choix aient été critiqués comme restant trop fidèles à une langue datant d'une époque révolue dans laquelle ne se reconnaissait plus la jeune génération, il contribua à faire revivre la langue littéraire catalane après une longue période de mise à l'écart durant la dictature du général Franco.

Dédié à tous les traducteurs qui ont permis la survie et la renaissance de la langue catalane, Less Translated Languages se veut à la fois un témoignage de la vitalité de la langue et de la culture catalanes ainsi qu'un plaidoyer en faveur de la diversité linguistique et culturelle comme facteur d'intensification des échanges entre les cultures.

Serge Marcoux

Université de Montréal, Montréal, Canada

\section{NOTES}

1. (NDLR) Le chapitre «Multilingualism in Europe» a été écrit alors que l'Union ne comptait encore que 15 États-membres. Elle compte maintenant 27 États-membres et 23 langues officielles (source: www.europa.eu/languages).

2. Translatability dans le texte original. 